\title{
ECG BIOMETRIC RECOGNITION WITHOUT FIDUCIAL DETECTION
}

\author{
Konstantinos N. Plataniotis, Dimitrios Hatzinakos, Jimmy K. M. Lee
}

\author{
Edward S. Rogers Sr. Department of Electrical And Computer Engineering \\ University Of Toronto
}

\begin{abstract}
Security concerns increase as the technology for falsification advances. There are strong evidences that a difficult to falsify biometric, the human heartbeat, can be used for identity recognition. Existing approaches address the problem by using electrocardiogram (ECG) data and the fiducials of the different parts of the heartrate. However, the current fiducial detection tools are inadequate for this application since the boundaries of waveforms are difficult to detect, locate and define. In this paper, an ECG biometric recognition method that does not require any waveform detections is introduced based on classification of coefficients from the Discrete Cosine Transform (DCT) of the Autocorrelation (AC) sequence of ECG data segments. Low false negative rates, low false positive rates and a $100 \%$ subject recognition rate for healthy subjects can be achieved for parameters that are suitable for the database.
\end{abstract}

\section{INTRODUCTION}

Biometric recognition promises airtight security by identifying an individual with its innate human features. The human fingerprint, voice, face, and iris are just a few features that are being used currently in biometric recognition systems. However, these features are still falsifiable. Some examples of falsification are 3D fingerprint and face models, audio recorders for voice playback, and contact lenses with copied iris features printed on. Recently, a few proposals [1-4] suggested the possibility of a new type of biometric that can be used and cannot be copied easily: the human heartbeat. The feature vectors, which are measured from different parts of the ECG signal, used in previous methods are listed in Table 1.

The validity of using ECG as a biometric recognition scheme is supported by the fact that physiological and geometrical differences of the heart in different individuals display certain uniqueness in their ECG signals [5]. Also in another recent study by PTB [6], the permanence characteristic of ECG pulses of a person was studied by noting that similarities of healthy subjects' pulses of different time intervals, from 0 to 118 days, can be seen when they are plotted one on top of each other at. These promising results suggest distinctiveness and long-term stability of the ECG as a biometric.

Previously proposed ECG recognition techniques [1-4] rely heavily on one major step to extract feature vectors: use of a fiducial detector. A fiducial detector is an automatic machine that marks the different components of an ECG signal. The problem is that these machines are built solely for the medical field in which only the approximate locations of fiducials are required for diagnostic purposes. Even if these detectors are accurate in identifying exact fiducial locations validated by cardiologists, there is no universally acknowledged rule for defining exactly where the wave boundaries lie [7].

TABLE 1: FEATURES FROM FOUR PREVIOUS EXPERIMENTS

\begin{tabular}{lll}
\hline QRS onset & QS interval & $\begin{array}{l}\text { R to T onset } \\
\text { interval }\end{array}$ \\
\hline P duration & QT interval & $\begin{array}{l}\text { R to T offset } \\
\text { interval }\end{array}$ \\
\hline QRS peak to peak & RS amplitude & $\begin{array}{l}\text { P wave pulse } \\
\text { width }\end{array}$ \\
\hline QRS duration & ST amplitude & $\begin{array}{l}\text { T wave pulse } \\
\text { width }\end{array}$ \\
\hline $\begin{array}{l}\text { R wave pulse } \\
\text { width }\end{array}$ & $\begin{array}{l}\text { QRS triangular } \\
\text { area }\end{array}$ & ST interval \\
\hline $\begin{array}{l}\text { S wave pulse } \\
\text { width }\end{array}$ & RQ interval & PQ interval \\
\hline ST slope & RS interval & PT interval \\
\hline $\begin{array}{l}\text { T wave min to } \\
\text { max amplitude }\end{array}$ & RP interval & $\begin{array}{l}\text { P onset to Q } \\
\text { interval }\end{array}$ \\
\hline ST amplitude & $\begin{array}{l}\text { R to P onset } \\
\text { interval }\end{array}$ & $\begin{array}{l}\text { S to T offset } \\
\text { interval }\end{array}$ \\
\hline RQ amplitude & $\begin{array}{l}\text { R to P offset } \\
\text { interval }\end{array}$ & \\
\hline RS slope & RT interval & \\
\hline \hline
\end{tabular}

An example is shown on Figure 1, where two pulses' P waves from the same person are plotted on top of each other with their $\mathrm{P}$ onset fiducial positions (provided by ECGPUWAVE $[8,9]$ ) marked. As seen, the two pulses' P onsets are marked very differently. Both the dashed pulse and the solid-lined pulse have two troughs at around $100 \mathrm{~ms}$ to $125 \mathrm{~ms}$ and $140 \mathrm{~ms}$ to $160 \mathrm{~ms}$. It is not clear if these small local minimums before the $\mathrm{P}$ wave are caused by noise or if it is part of the $\mathrm{P}$ wave. There is no definite 

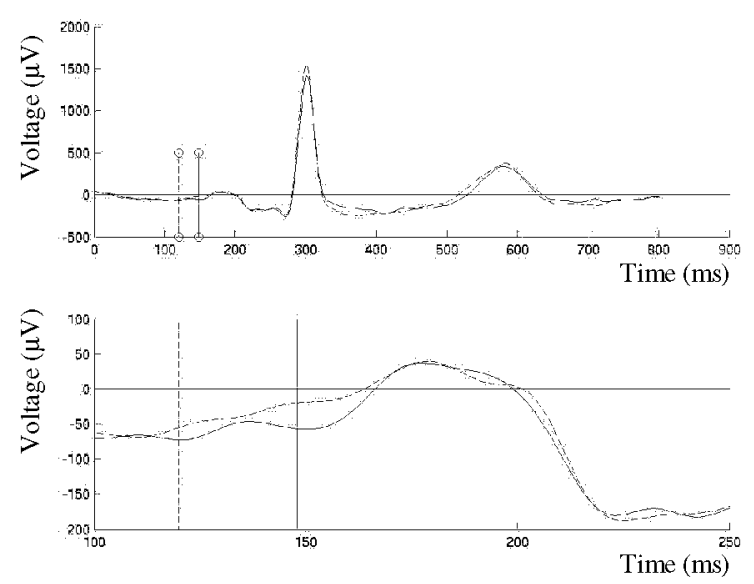

Figure 1: (Top) Two pulses from a subject of the PTB database [8] plotted on top of each other, aligning at the $\mathrm{R}$ peak, which happens to be also the $P$ wave peak in this case due to the same heartrates. (Bottom) Zoomed in view at time $100 \mathrm{~ms}$ to $250 \mathrm{~ms}$, showing the $\mathrm{P}$ wave onset markers from ECGPUWAVE [8,9]

answer which of the two areas should be marked as the onset of the $\mathrm{P}$ waves.

In contrast to most existing methods, the solution proposed in this paper does not require a fiducial detector. Rather, this method is dependent on estimating and comparing the significant coefficients of the Discrete Cosine Transform (DCT) of the windowed autocorrelations (AC) of heartbeat records. As such, it is insensitive to heart rate variations, simple and computationally efficient. It also does not require pulse synchronization. In addition, it exhibits better identification performance as demonstrated by computer simulations.

\section{NEW APPROACH AND METHODOLOGY (AC/DCT)}

Given two ECG records of arbitrary length $N$, each containing a multiple of heartbeat periods, the new approach proceeds as follows: 1) the ECG records are first conditioned by filtering, for DC and noise reduction, 2) the normalized autocorrelation function of each record is estimated over a window of $M$ autocorrelation lags, 3) the DCT of the windowed autocorrelation is calculated and the first $C$ number of significant coefficients are selected, and 4) the selected DCT coefficients of the two records are used for identification and classification. We refer to this method as the AC/DCT method. Data from different stages of this process are depicted in Figure 2.

\subsection{ECG conditioning}

As with the other methods [1-4], first the ECG traces are filtered to remove noise. Pass-band filtering (0.5 to $40 \mathrm{~Hz})$ as in [3] is used to filter out unwanted noise and artifacts while preserving heartbeat fiducial features.

Following filtering, instead of extracting individual ECG pulses, the AC/DCT method considers a window of the ECG trace data of arbitrary length and origin. The only requirement imposed is that the data window of length $N$ is longer than the underlying average heart beat rate so in normal situations it can contain multiple pulses. Also, it is not necessary for it to contain all complete heartbeat periods. Thus, contrary to most existing heartbeat biometric identification methods, this method introduces two main advantages: a) exact heart rate detection, which may vary between different records or even within the same record of data, is not required; and b) no synchronization of heartbeat pulses is necessary. These contribute to the appealing computational simplicity and robustness of the proposed approach.

Depending on the sampling rate, the length of the data window may vary. This offers a compromise between representing a multiple of unique and varying subject characteristics and computational complexity. As a rule of thumb, based on our experimentation, a data window of 10 seconds has been found to be a good choice for the AC/DCT method [10].

Let an ECG window of length $N$ be $x[i]$. $N$ would be the number of samples inside the window. For a database with a sampling rate of $1000 \mathrm{~Hz}$ as in the PTB database $[8,11]$ and using the typical window length value of 10 seconds, $N$ would be equal to 10000 samples.

\subsection{Autocorrelation (AC)}

The autocorrelation is applied to accomplish the following objective: to blend in all samples in the ECG window to a sequence of sums of products so that the actual locations of the fiducials will not be required to be explicitly found. The estimated and normalized AC formula used for this approach is shown here:

$$
\hat{R}_{x x}[m]=\frac{\sum_{i=0}^{N-|m|-1} x[i] x[i+m]}{\hat{R}_{x x}[0]}
$$

where $x[i]$ is the windowed ECG and $x[i+m]$ is the timeshifted version of the windowed ECG with a time lag of $m$ $=0,1, \ldots,(M-1) ; M<<. M$ is a parameter that is to be chosen and this will be discussed later. The reader should note that it does not matter if the estimation is biased or unbiased because the division with the maximum value, $\hat{R}_{x x}[0]$, cancels out the biasing factor. 
a) 5 seconds of ECG from subject $A$


e) 400 AC Coefficients of A Time $(\mathrm{ms})$
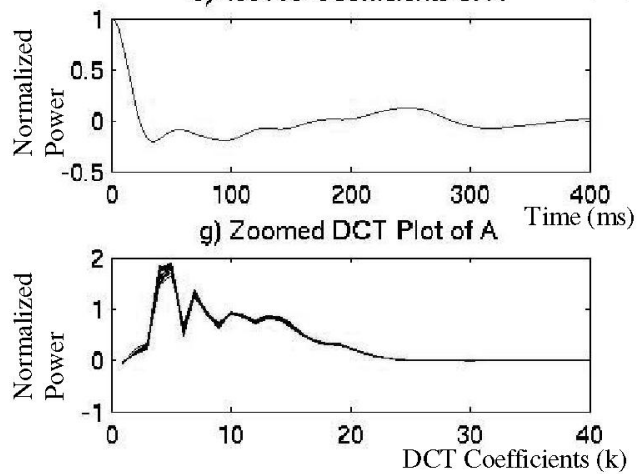

b) 5 seconds of ECG from subject $B$
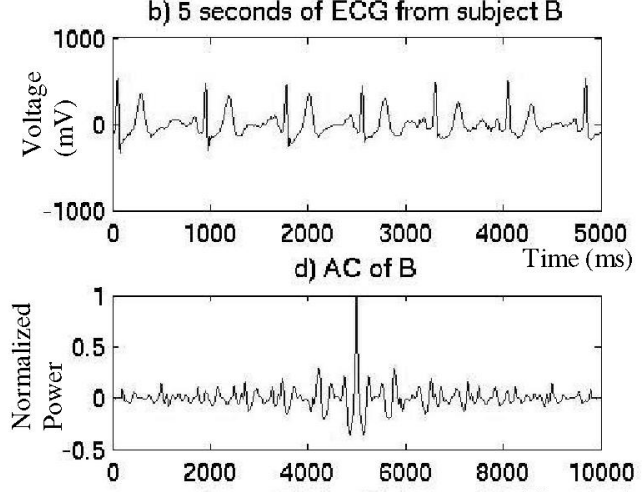

f) 400 AC Coefficients of B Time (ms)
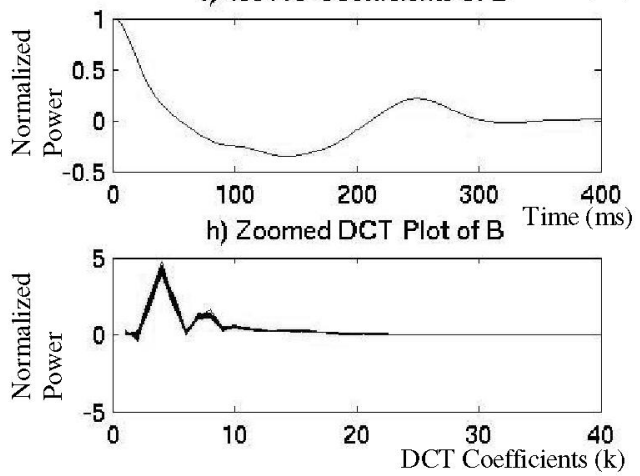

Figure 2: ( $a$ and b) 5 seconds window of ECG from two subjects of the PTB database, subject A and B. Notice that in subject B the window chops off part of a pulse. ( $c$ and d) The normalized autocorrelation sequence of $\mathrm{A}$ and $\mathrm{B}$. (e and $\mathrm{f}$ ) Zoomed in to $400 \mathrm{AC}$ coefficients from the maximum. ( $\mathrm{g}$ and $\mathrm{h}$ ) DCT of the $400 \mathrm{AC}$ coefficients from 10 ECG windows including the one on top. Notice that the same subject has similar shapes.

A typical ECG pulse consists of mainly three high amplitude waveforms: the P complex, the QRS complex, and the $\mathrm{T}$ complex. These complexes are the main contributors to this sum when the autocorrelation coefficients are calculated on a section of the ECG signal.

The utilization of a finite number of the autocorrelation lags of ECG data records for biometric identification has been motivated by the following observations:

a) ECG records have a repetitive structure but they are not periodic. Both pulse cycle lengths as well as relative distances between $\mathrm{P}, \mathrm{QRS}$ and $\mathrm{T}$ complexes vary with respect to time of ECG acquisition, subject's physical state, mental state, and many other factors. The autocorrelation provides an automatic, shift invariant accumulation of similarity features over multiple signal cycles and therefore, we anticipate that it will be a better representative of the unique characteristics of a given ECG.

b) For a given subject, the QRS complex exhibits the least variability in time under different measurement conditions [3]. Therefore, the first $M$ autocorrelation lags corresponding to a maximum lag of approximately equal to the length of a QRS complex. This should provide more robust representation of the subject's heartbeat characteristics.

Our expectations are confirmed by the results of Figure 3, which shows the $\hat{R}_{x x}[m]$ obtained from different ECG windows of the same subject from two different records in the PTB database $[8,11]$ taken at a different time.

\subsection{Discrete cosine transform (DCT)}

The DCT is applied to the AC coefficients for dimensionality reduction. After DCT is performed, the number of important coefficients is reduced even more because a lot of the DCT coefficients will become near-zero values. This is a result of the energy compaction property of the DCT transform. Therefore, assuming we take an $M$ point DCT, only $C \ll M$ DCT coefficients will be significant as can be easily seen from Figure $2 \mathrm{~g}$ and $2 \mathrm{~h}$. The $C$ first coefficients of the DCT form the feature vector of the proposed AC/DCT biometric identification method. 




Figure 3: AC sequences of two different records taken at different times from the same subject of the PTB database $[8,11]$. The sequence with the same record is plotted with the same shade.

\section{PERFORMANCE EVALUATION}

To provide a comparative evaluation of the discriminating value of the proposed feature vector, we have carried out experiments that contain the two distance metrics described below. Furthermore, the false negative and false positive rates of these metrics will be evaluated as well.

\subsection{Normalized Euclidean distance}

The normalized Euclidean distance $D$ between two feature vectors $\mathbf{x}_{\mathbf{1}}$ and $\mathbf{x}_{\mathbf{2}}$ is defined as

$$
D\left(\mathbf{x}_{1}, \mathbf{x}_{2}\right)=\frac{1}{C} \sqrt{\left(\mathbf{x}_{1}-\mathbf{x}_{2}\right)^{T}\left(\mathbf{x}_{1}-\mathbf{x}_{2}\right)}
$$

where $C$ is the dimension of the feature vectors, which is the number of DCT coefficients in this experiment. This factor is there to make comparisons fair for different dimensions $\mathbf{x}$ might have.

\subsection{Normalized Gaussian log likelihood}

If a Gaussian distribution is assumed, the Gaussian $\log$ likelihood function can be used for a distance metric. Assuming that the prior probability for the occurrences of each subject is the same, and assuming that the variances for each subject are the same, the normalized Gaussian $\log$ likelihood function is defined in the following:

$$
L_{i}(\mathbf{x})=-\frac{1}{2 C}\left(\mathbf{x}-\overline{\mathbf{x}}_{i}\right)^{T} S^{-1}\left(\mathbf{x}-\overline{\mathbf{x}}_{i}\right)
$$

where $\overline{\mathbf{x}}_{i}$ are the respective sample mean vectors for subject $i$, and $S$ is the estimated overall covariance matrix for the whole database. The resultant $L_{i}$ would indicate the score for $\mathbf{x}$ belonging to subject $i$. The higher the score, the higher the possibility of $\mathbf{x}$ to be a feature vector from subject $i$. The highest $L_{i}$ value indicates that the particular $\mathbf{X}$ is most likely belonging to subject $i$. Since different feature vector dimensions will give different mean magnitudes, the factor $C$ is there again to allow fair comparisons.

First, $\mathbf{x}_{1}$ and $\mathbf{x}_{\mathbf{2}}$ were calculated from two different data records of the same subject taken at different times. Performing a large number of such trials the mean and standard deviation of the two metrics were calculated to provide a statistical description of matching same subject records. Then, $\mathbf{x}_{1}$ and $\mathbf{x}_{2}$ were calculated from two different data records from different subjects. The mean and standard deviation (SD) of the two metrics in this case provide a statistical description of discrimination between different subject records.

Figures 4 and 5 show plots of mean and SD for the proposed AC/DCT method compared with the
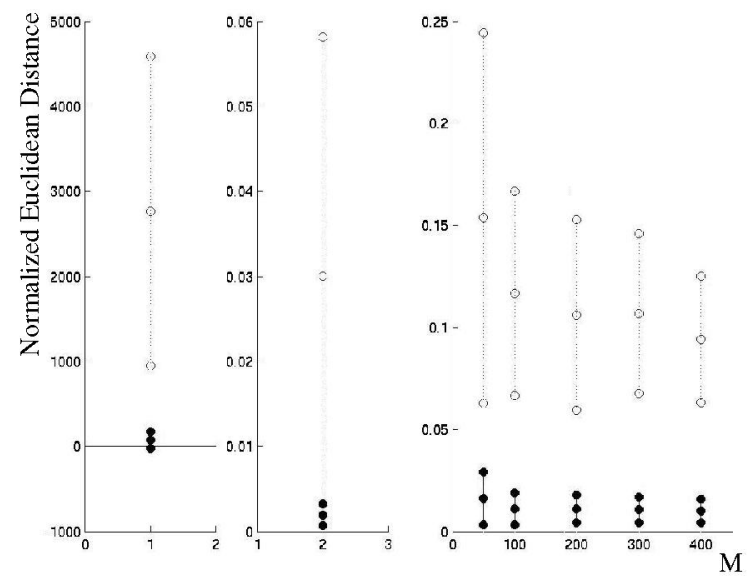

Figure 4: Normalized Euclidean distance plots of different methods. From the left: [1], [3], AC/DCT with $\mathrm{M}=50,100,200,300,400$.

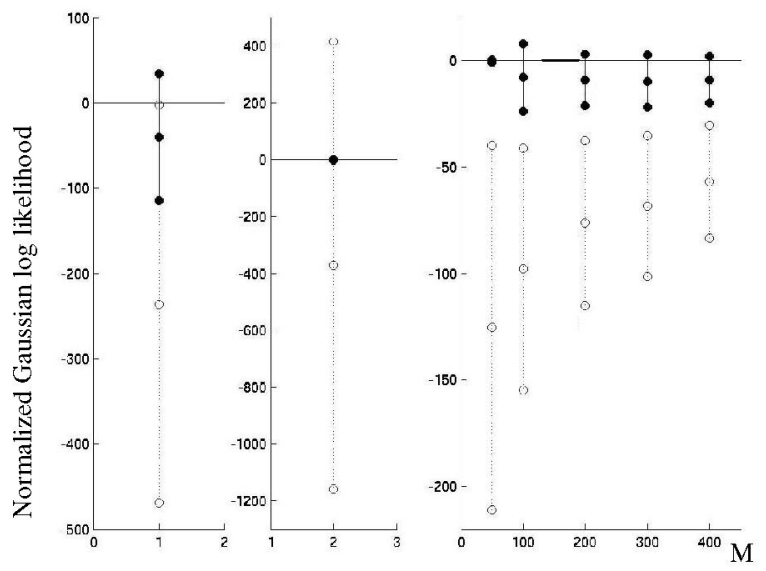

Figure 5: Normalized Gaussian log likelihood plots of different methods. From the left: [1], [3], AC/DCT with $\mathrm{M}=50,100,200$, 300,400 . 
corresponding values of previous methods using different implementation parameters. The experiment was performed with data from 14 healthy subjects with multiple records obtained from the PTB database [8,11]. An ECG window duration of 10 seconds ( $N=10000$ samples) was used for all experiments. Each record has a length of 100 seconds; therefore there are 10 windows for each subject.

An approximate total of 14000 pulses exist in the database. The number of DCT coefficients, $C$, is set differently for each $M$ and it is the coefficient number where the value drops belong $0.2 \%$ of the highest value in the DCT sequence for metric described by (2). Due to the use of sample variance in (3), a different rule for setting $C$ relative to the different $M$ values is required. Experiment shows that choosing the first coefficient with an amplitude value of about $0.2 \%$ of the highest DCT coefficient at the decaying tail of the DCT sequence as the cutoff value yields the best results.

For comparison purposes, results from using the feature vectors of the methods in [1] and [3] have been also included. It is clearly observed from Figures 4 and 5 that the feature extraction of the AC/DCT method provides higher separability and discriminating capability than the other methods. Hence, this is indicative that one can expect biometric identification performance improvements with the proposed method.

\subsection{False negative (FN) and false positive (FP) performance}

To find FN and FP, we assume that the estimated metrics $\left(D\right.$, or $L_{i}$ ) have a Gaussian distribution. This assumption is valid because the results of these comparisons are supposedly coming from either the same subject or different subject and thus, they are random variables defined on either one of the two respective probability spaces. In addition, since the determination of any $D$ or $L_{i}$ is independent at each trial, it is feasible to assume that the $D$ 's and $L_{i}$ 's are i.i.d. random variables. Therefore, they have Gaussian distribution from the central limit theorem.

Utilizing the Gaussian assumption, the mean and standard deviation for same and different subjects obtained earlier, $F N$ and $F P$ are calculated by first finding the intersection $t$, between the same-subject's Gaussian PDF and the different-subject's Gaussian PDF. After finding $t$, FN and FP can be found by (4) and (5) for the case of the normalized Gaussian log likelihood metric:

$$
\begin{aligned}
& F N_{\text {likelihood }}=P_{\text {same }}(-\infty<x<t) \\
& F P_{\text {likelihood }}=P_{\text {different }}(t<x<\infty)
\end{aligned}
$$

where $x$ is the Gaussian random variable with the mean and SD of the corresponding subject groups. Similar calculations can be applied to the normalized Euclidean distance also by reversing the ranges in the probabilities.

\section{CLASSIFICATION PERFORMANCE}

To estimate the classification performance of the proposed method, which is the ability to correctly classify (assign) a given pulse record to the corresponding subject, the AC/DCT method was applied to two different records of the 14 healthy subjects from the PTB database. The estimated DCT feature vector for each subject in record 1 was compared to the DCT feature vectors of all subjects in record 2 and the best match was determined. The corresponding results are given in Tables 2 and 3 for the two distance metrics described above.

TABLE 2: RESULTS FROM CLASSIFICATION EXPERIMENTS WITH TWO PTB DATABASES USING NORMALIZED EUCLDEAN DISTANCE

\begin{tabular}{rrrrrc}
\hline \hline$M$ & $C$ & $\begin{array}{l}F N \text { (same } \\
\text { database) }\end{array}$ & $\begin{array}{l}F P \text { (same } \\
\text { database) }\end{array}$ & $\begin{array}{l}\text { Window } \\
\text { Recognition } \\
\text { Rate }\end{array}$ & $\begin{array}{l}\text { Subject } \\
\text { Recognition } \\
\text { Rate }\end{array}$ \\
\hline 50 & 5 & 0.0106 & 0.1177 & $116 / 140$ & $13 / 14$ \\
\hline 100 & 11 & 0.0049 & 0.0435 & $119 / 140$ & $12 / 14$ \\
\hline 180 & 13 & 0.0061 & 0.0587 & $132 / 140$ & $13 / 14$ \\
\hline 200 & 17 & 0.0052 & 0.0490 & $129 / 140$ & $13 / 14$ \\
\hline 300 & 19 & 0.0028 & 0.0224 & $126 / 140$ & $13 / 14$ \\
\hline 400 & 26 & 0.0022 & 0.0146 & $125 / 140$ & $13 / 14$ \\
\hline \hline
\end{tabular}

From the results in Table 2, we observe that the best performance has been obtained for the Normalized Euclidean Distance when $M=180 \mathrm{~ms}$ (shown shaded in Table 2), which corresponds approximately to the length of the QRS complex as to our expectations explained in section 2.2 .

The results in Table 3 show that it is possible to have perfect subject recognition using the Gaussian log likelihood. With a larger $C$, three cases for $M$ gave perfect subject recognition rates and two cases gave perfect window recognition rates (shown shaded). The assumption of Gaussian distribution of the ECG windows turns out to be helpful in the classification performance, yielding high recognition rates.

TABLE 3: RESULTS FROM CLASSIFICATION EXPERIMENTS WITH TWO PTB DATABASES USING NORMALIZED GAUSSIAN LOG LIKELIHOOD

\begin{tabular}{rrlccc}
\hline \hline$M$ & $C$ & $\begin{array}{l}F N(\text { same } \\
\text { database) }\end{array}$ & $\begin{array}{l}F P(\text { same } \\
\text { database) }\end{array}$ & $\begin{array}{l}\text { Window } \\
\text { Recognition } \\
\text { Rate }\end{array}$ & $\begin{array}{l}\text { Subject } \\
\text { Recognition } \\
\text { Rate }\end{array}$ \\
\hline 50 & 21 & $5.2716\left(10^{-4}\right)$ & 0.0529 & $136 / 140$ & $14 / 14$ \\
\hline 100 & 27 & $3.6657\left(10^{-4}\right)$ & 0.0367 & $140 / 140$ & $14 / 14$ \\
\hline 200 & 44 & $1.6759\left(10^{-4}\right)$ & 0.0194 & $140 / 140$ & $14 / 14$ \\
\hline 300 & 63 & $7.4346\left(10^{-5}\right)$ & 0.0122 & $130 / 140$ & $13 / 14$ \\
\hline 400 & 55 & $6.5794\left(10^{-5}\right)$ & 0.0085 & $130 / 140$ & $13 / 14$ \\
\hline \hline
\end{tabular}

Note that $M$ values over $200 \mathrm{~ms}$ give poor performances. This could be due to the inclusion of positional data from 
the $\mathrm{T}$ wave at these $M$ values since the $\mathrm{T}$ wave moves relative to the QRS complex at different heartbeat rates. It is not useful information to be included.

Also note the very low false negative rates resulting from the AC/DCT method. This suggests that this method could be applied to biometric recognition scenarios with high FN costs.

\section{CONCLUSIONS AND FUTURE IMPROVEMENTS}

The results of the experiment show that it is possible to perform ECG biometric recognition without the use of PQRST fiducial detection. The proposed AC/DCT method provides an efficient, robust, and computationally efficient heartbeat biometric identification technique. The method offers significant computational advantages compared to existing approaches with better or comparable classification performance. An additional advantage of this technique is that is not limited to ECG records only; it is general enough that it is applicable to other modes of heartbeat recordings, such as acoustic signals, since it does not depend on ECG specific characteristics. Further experimentation however, is needed to determine the effectiveness of the method with non-healthy subjects.

\section{REFERENCES}

[1] T. W. Shen, W. J. Tompkins, and Y. H. Hu, "One-Lead ECG for Identity Verification," Proceedings of the Second Joint EMBS/BMES Conference. Houston TX, USA, 2001

[2] Lena Biel, et al., "ECG Analysis: A New Approach in Human Identification," IEEE Transactions on Instrumentation and Measurement, Vol. 50, No. 3, 2001.

[3] Steven A. Israel, et al., "ECG to identify individuals," Pattem Recognition Society 2005. Elsevier Ltd., USA, 2004.

[4] Steven A. Israel, et al., "Fusing Face and ECG for Personal Identification," Proceedings of the 32nd Applied Imagery Pattem Recognition Workshop (AIPR' 03), 2003.

[5] R. Hoekema, G.J.H. Uijen, A. van Oosterom, "Geometrical aspect of the interindividual variability of multilead ECG recordings", IEEE Trans. Biomedical. Engineering Vol. 48, pp. 551-559, 2001

[6] G. Wuebbeler, et al., Human Verification by Heart Beat Signals, Working Group 8.42, Physikalisch-Technische Bundesanstalt (PTB), (http://www.berlin.ptb.de/8/84/842/BIOMETRIE/842biometriee.html)

[7] J. P. Martínez et al., "A Wavelet-Based ECG Delineator: Evaluation on Standard Databases," IEEE Transactions on Biomedical Engineering, Vol. 51, No. 4, pp. 570-581, April 2004

[8] A. Goldberger, et al., "PhysioBank, PhysioToolkit, and PhysioNet: Components of a New Research Resource for Complex Physiologic Signals," Circulation, Vol. 101, No. 23, pp. e215-e220 [Circulation Electronic Pages; http://circ.ahajournals.org/cgi/content/full/101/23/e215], 2000

[9] Laguna P., Jané R., Bogatell E., and Anglada D. V., "QRS detection and waveform boundary recognition using ecgpuwave," 2002 (http://www.physionet.org/physiotools/ecgpuwave/)

[10] J. K. M. Lee, ECG Feature Extraction without Fiducial Detection: Applications to ECG Biometric Recognition, M. A. Sc. dissertation, Faculty of Applied Science and Engineering, University of Toronto, 2006

[11] Michael Oeff, et al., The PTB Diagnostic ECG Database, National Metrology Institute of Germany, (http://www.physionet.org/physiobank/database/ptbdb/) 